\title{
Aligning molecular studies of mycorrhizal fungal diversity with ecologically important levels of diversity in ecosystems
}

\author{
Ian R Sanders ${ }^{1,3}$ and Alia Rodriguez ${ }^{2}$ \\ ${ }^{1}$ Department of Ecology and Evolution, University of Lausanne, Lausanne, Switzerland and ${ }^{2}$ Biology \\ Department, Faculty of Science, National University of Colombia, Ciudad Universitaria, Bogotá, Colombia
}

\begin{abstract}
Arbuscular mycorrhizal fungi (AMF) occur in the roots of most plants and are an ecologically important component of the soil microbiome. Richness of AMF taxa is a strong driver of plant diversity and productivity, thus providing a rationale for characterizing AMF diversity in natural ecosystems. Consequently, a large number of molecular studies on AMF community composition are currently underway. Most published studies, at best, only address species or genera-level resolution. However, several experimental studies indicate that variation in plant performance is large among plants colonised by different individuals of one AMF species. Thus, there is a potential disparity between how molecular community ecologists are currently describing AMF diversity and the level of AMF diversity that may actually be ecologically relevant. We propose a strategy to find many polymorphic loci that can define within-species genetic variability within AMF, or at any level of resolution desired within the Glomermycota. We propose that allele diversity at the intraspecific level could then be measured for target AMF groups, or at other levels of resolution, in environmental DNA samples. Combining the use of such markers with experimental studies on AMF diversity would help to elucidate the most important level(s) of AMF diversity in plant communities. Our goal is to encourage ecologists who are trying to explain how mycorrhizal fungal communities are structured to take an approach that could also yield meaningful information that is relevant to the diversity, functioning and productivity of ecosystems.
\end{abstract}

The ISME Journal (2016) 10, 2780-2786; doi:10.1038/ismej.2016.73; published online 29 April 2016

\section{Introduction}

Arbuscular mycorrhizal fungi (phylum Glomeromycota) are, without question, an ecologically important component of soils, forming symbioses with $~ 200000$ plant species in all the major biomes (Davison et al., 2015; van der Heijden et al., 2015). Arbuscular mycorrhizal fungi improve plant $\mathrm{P}$ acquisition and also other soil nutrients (Harrison, 1999; Hodge et al., 2001). Arbuscular mycorrhizal fungi exert a strong influence on plant community structure, plant diversity and ecosystem function (van der Heijden et al., 1998a, b).

Despite their ecological importance, surprisingly little is known about basic AMF ecology, their biogeography and the factors governing the structure of AMF communities. As with most soil microbiota, molecular techniques to measure diversity have

Correspondence: IR Sanders, Department of Ecology and Evolution, University of Lausanne, Biophore Building, Lausanne 1015, Switzerland.

E-mail: ian.sanders@unil.ch

${ }^{3}$ These authors contributed equally to this work.

Received 18 November 2015; revised 10 March 2016; accepted 23 March 2016; published online 29 April 2016 allowed ecologists to study AMF communities in a way that was not possible a few years ago. Most of the studies on AMF diversity evaluate variation in DNA sequences derived from amplicons of specific targeted genes or regions of the genome; sometimes referred to as a meta-genomic approach. Despite this worthwhile pursuit, in this Perspectives article, we argue that in order to understand the role of AMF in shaping plant communities, molecular data need to be aligned with relevant levels of AMF diversity. Here we explain why approaches might currently not be aligned and we propose some possible solutions that may be more ecologically relevant for understanding how the diversity of AMF influences plant ecology.

In a first step, researchers using molecular approaches to document AMF diversity should consider findings from experimental studies that identify which are the ecologically relevant levels of AMF diversity that influence plant ecology. In a second step, these scientists then need to develop molecular approaches to allow the measurement of those ecologically relevant levels of AMF diversity. In a third step, researchers need to then measure ecologically relevant levels of AMF diversity in 
nature or in experiments that will help to pinpoint the role of AMF diversity in plant ecology and ecosystem functioning. In order to understand the problem, we first need to consider the ecological rationale for measuring AMF diversity in communities and also understand the way in which molecular techniques are currently used to measure such diversity.

\section{The rationale for measuring AMF diversity}

The measurement of AMF diversity in ecosystems is carried out for many different reasons. The most fundamental reason is to find out more about the basic ecology of AMF. New knowledge about AMF ecology has being successfully uncovered with the application of high-throughput sequencing-based techniques, with exciting and intriguing results (Box 1). For this reason, this Perspectives article by no means represents a criticism of previously published molecular studies or their important findings.

We assume that a driving force in documenting AMF diversity resides in their ecological importance, especially as increasing richness of AMF taxa was shown to exert a positive effect on plant community productivity, acquisition of phosphate and plant diversity (van der Heijden et al., 1998b). Thus, a major ecological goal should be to better understand the importance of AMF diversity in structuring plant communities and to understand how it contributes to the functioning of 'healthy ecosystems'. Given the rapid climatic changes to ecosystems, it is important to understand how any environmentally induced changes in AMF diversity might influence plant communities, other microbial communities and the functioning of ecosystems. Finally, an important goal should be to establish whether given levels of AMF diversity are indicators of healthy or unhealthy ecosystems. However, in order to establish such relationships molecular approaches are needed that actually target the ecologically relevant levels of AMF diversity.

\section{Finding ecologically relevant levels of AMF diversity}

There are numerous published examples of apparent differential effects of AMF species on plant growth. Here we refer to AMF species as morphologically recognisable taxa (based on spore morphology) from which DNA sequencing has allowed the construction of a well-supported phylogeny (Krüger et al., 2012). Because many studies have shown large differences in growth among plants inoculated with different AMF species, this has lead researchers to focus on AMF species or genera differences as probably representing a functionally important level of AMF diversity.
Box 1 Ecological advances made in our understanding of AMF communities using meta-genomics

The identification and quantification of different AMF taxa from roots or soil using DNA-based techniques is essential to understand AMF community and population structure. Some more notable studies are mentioned here.

Early indications from molecular-based studies of AMF communities revealed that a certain amount of selectivity of AMF species by certain plant functional groups exists and that AMF assemblages in boreo-nemoral forests are non-random (Davison et al., 2011); an ecological question that had remained unanswered for many years. Using a very different analytical approach known as an evolutionary placement algorithm, potato crops in a wide range of distantly distributed sites across Peru and Ecuador contained a remarkably similar AMF species assemblage in their roots (Senés-Guerro and Schussler, 2015). Such similarities among sites were not so easy to determine with more conventional clustering techniques.

AMF communities have also been shown to be highly dynamic, with communities changing both in season and by year (Davison et al., 2011; Cotton et al., 2015). Land use has also been shown as a likely influence on AMF community composition as different AMF phylogenetic structure was observed in soils with different land-use practices (Moora et al., 2014).

Studies have also started to look in closer detail at the relationship between AMF community diversity and plant diversity and productivity. A very original approach using 454 pyro-sequencing showed that AMF richness is more closely correlated with below-ground plant species richness (which includes DNA sequences from the seed bank and from plants that might not be growing at the season of sampling) and above-ground plant species richness and that AMF richness is negatively correlated with plant below-ground biomass (Hiiesalu et al., 2014).

Finally, studies of AMF community composition using 454 pyro-sequencing are opening up our knowledge about AMF diversity and distribution at different spatial scales. A core group of generalist AMF species occurred at most of the 50 German grassland sites studied in one region (Vályi et al., 2015). A study of fungal and AMF community diversity across the Vaudois alps in Switzerland, with a very steep altitudinal gradient, included over 200 sites (Pellissier et al., 2014). Using a highly robust hierarchical clustering technique known as DBC 454 (Pagni et al., 2013), Pellissier et al. (2013) were able to demonstrate that an area of $\sim 16 \mathrm{~km}^{2}$ was required to encompass most of the AMF diversity found in the region.

Finally, a study of global AMF distribution ascribing AMF 454 sequence reads to virtual taxa, revealed that the majority of taxa are distributed over a very wide range, with $93 \%$ of taxa occurring on more than one continent and a highly surprising $34 \%$ occurring on all six continents (Davison et al., 2015). This is highly unusual as it was previously thought that AMF have a very limited dispersal capacity. Population genomics on individual AMF species could help to uncover the mystery of how AMF taxa have been able to disperse over long distances and attain a seemingly ultra-wide niche.

We suggest that placing the ecological importance on AMF species differences may well be misleading. Most studies showing significant differential AMF isolate effects on plant growth have used only one isolate per AMF species. The underlying assumption in such studies is that one isolate is representative of the species. However, including several different isolates of each AMF species can lead to very different conclusions. We illustrate this with a hypothetical experiment, where two AMF species 
and three isolates of each AMF species were used to inoculate one plant species and the variation in plant growth among the treatments was quantified (Figure 1). Let us imagine that variation in plant growth was significantly different among all isolates, but that the majority of the variation in plant growth was found among plants inoculated with different isolates of the same AMF species (Figure 1a). Indeed in Figure 1a, the mean plant growth response to the two AMF species is very similar. If we repeat the experiment, but randomly choosing only one isolate per AMF species, we would likely observe that the two different AMF species resulted in differential plant growth and may wrongly conclude that the important level of variation in plant growth is determined by different AMF species (Figure 1b). The experiment shown in Figure $1 \mathrm{~b}$ has been performed many times, but very few experiments have followed the design in Figure 1a. The other extreme is where no significant differences occurred in plant growth among plants inoculated with the different isolates of the same AMF species (Figure 1c). Thus, the results of the second experiment indeed represent differences in plant growth caused by interspecific AMF differences (Figure 1d).

Although the experiment in Figure 1a has rarely been performed, evidence from two published studies suggests that variation in effects of AMF on plant growth can be larger among plants inoculated with AMF of the same species than inoculation with different species. Munkvold et al. (2004) inoculated cucumber (Cucumis sativus) with four AMF species and several isolates of each species. Large significant intraspecific variation in AMF was observed in plant growth, fungal $\mathrm{P}$ uptake, plant shoot $\mathrm{P}$ uptake and extra-radicle AMF hyphal length (Munkvold et al., 2004). In a recent study, alfalfa (Medicago sativa) was inoculated with several different isolates of each of 10 AMF species (Mensah et al., 2015). Again, very large growth differences were reported among plants inoculated with different isolates of the same AMF species, especially in terms of root biomass. Thus, the results from these experiments are similar to those in the hypothetical experiment depicted in Figure 1a. Randomly choosing one isolate from each

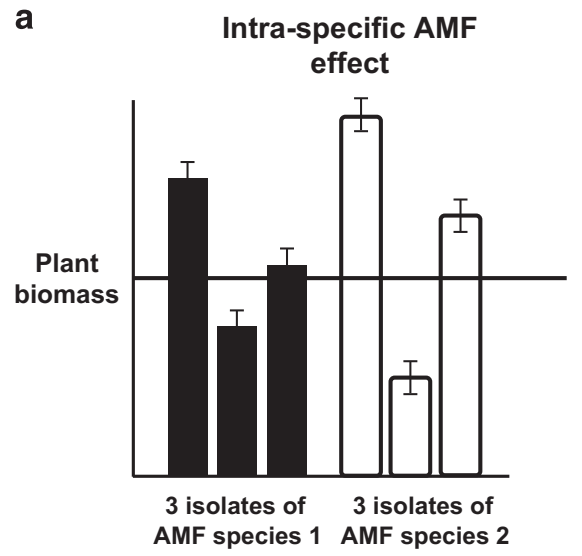

c
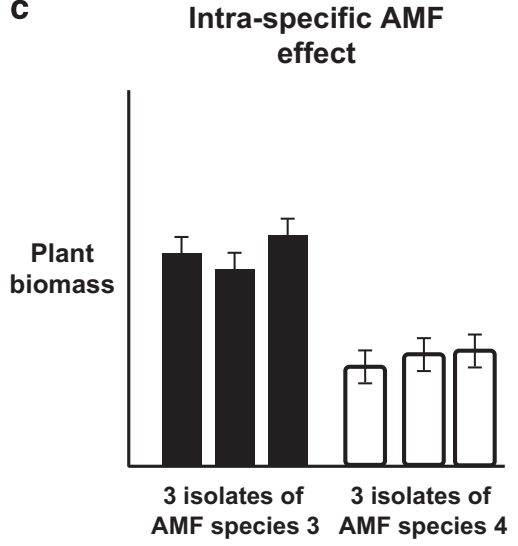

b

False inter-specific AMF efect

d

True inter-specific AMF efect
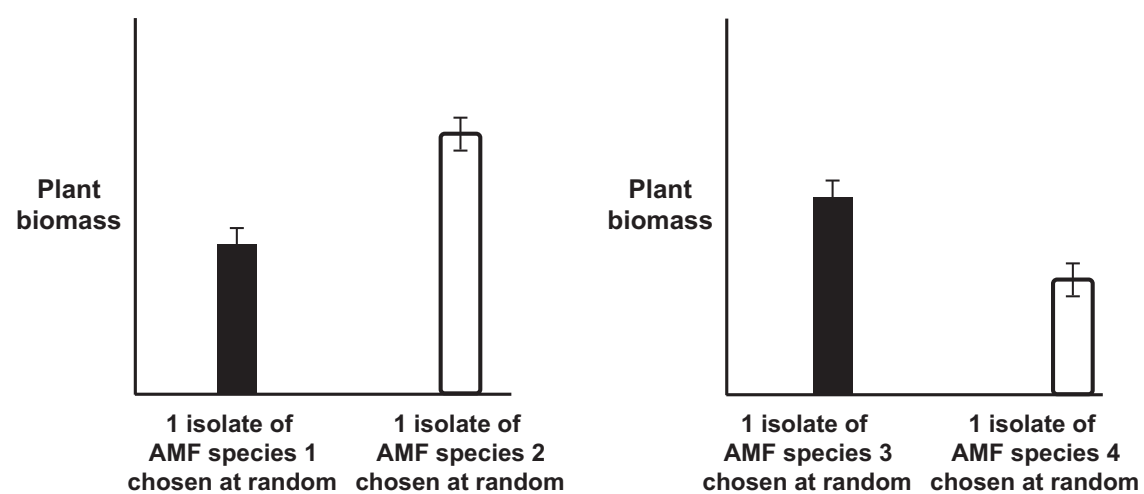

Figure 1 Results of a hypothetical experiment where the effects of different AMF isolates and species on plant growth are tested. (a) In experiment 1, intraspecific variation in AMF isolates on plant growth is observed but the mean response of the plants (shown by the horizontal line) to each AMF species is the same. (b) If one isolate of each AMF species used in experiment 1 would be selected at random then results appear to resemble an AMF species effect on plant growth. (c) In a second experiment, interspecific effects of AMF on plant growth are observed. (d) If one isolate of each AMF species would be selected at random for experiment 2 then results would represent a true AMF species effect on plant growth. 
AMF species for a second experiment would have a high likelihood of giving the results depicted in Figure 1b.

The studies by Munkvold et al. (2004) and Mensah et al. (2015) indicate that intraspecific variation could potentially be the most ecologically important level of variation in AMF. However, there are at least two caveats. First, the fungi originated from different places. Thus, the studies do not provide a measure of intraspecific AMF effects on plant growth from a true population. One study shows significant intraspecific AMF effects on plant growth among isolates from one population. However, there was no comparison of interspecific versus intraspecific AMF effects (Koch et al., 2006). Second, so far, studies of intraspecific AMF effects on plant growth have been conducted with only one plant species. More plant species are required in such experiments in order to establish whether there are plant species $\times \mathrm{AMF}$ intraspecific isolate interactions. To measure the importance of intraspecific variation in AMF as potential drivers of diversity and productivity in plant communities would require experiments like that of van der Heijden et al. (1998b) to be conducted with different levels of richness of different isolates of AMF species, with a true among-AMF species comparison.

Although intraspecific genetic variation in AMF may be ecologically very important, current molecular approaches to documenting AMF diversity completely ignore this level of diversity. Alternative molecular strategies to documenting genetic diversity in AMF communities are greatly needed to encompass this diversity.

\section{Levels of resolution in AMF diversity afforded by current molecular approaches}

The ecological relevance of generated meta-genomic data on AMF must be governed by the resolution afforded by the methodology. That resolution largely depends on the region of the genome that is sequenced and the subsequent treatment of the data; particularly how the data are clustered and matched to available reference databases.

Current approaches and challenges of using metagenomics techniques for studying AMF communities were recently reviewed (Hart et al., 2015) and some of the notable advances in understanding AMF ecology afforded by these approaches is shown in Box 1. The majority of AMF meta-genomic studies have focussed on regions of the ribosomal DNA; the small subunit rRNA (SSU), the internal transcribed spacer or the large subunit (LSU). In sequence data sets, subsequent clustering of rDNA reads is made into operational taxonomic units either with or without comparison to a reference database or the identification of sequences to virtual taxa (VT; Öpik et al., 2010). Virtual taxa are phylogenetically resolved groups of sequences that give taxonomic resolution similar to morphologically recognised species, but only for some families (Öpik et al., 2013). At present, the largest and most frequently updated database is MaarjAM containing AMF SSU sequences that can be used to define VT (Öpik et al., 2010). Many groups use this database as the standard reference. Using the VT approach, or robust hierarchical clustering methods such as DBC454 (Pagni et al., 2013), it is possible to obtain clusters or identities approaching the species level at best. Thus, current methods for studying AMF diversity do not encompass the level of AMF diversity that might be more ecologically relevant (as described in Figure 1) and, therefore, may not be appropriate for studying the role of AMF diversity in ecosystem ecology.

Establishing and curating a database such as MaarjAM requires a very large effort. When databases already exist, researchers can contribute additional sequences, allowing an evolving resource. Finding new informative markers and developing similar resources with different levels of resolution is such a large undertaking. It is, therefore, very tempting for any groups lacking substantial financial resources to use existing databases and approaches for studies on AMF diversity without questioning the ecological relevance of the data generated.

\section{Aligning molecular approaches with ecologically relevant levels of AMF diversity}

Because current meta-genomics approaches to documenting AMF communities may be misaligned with measurements of ecologically relevant levels of AMF diversity, alternative molecular approaches are required that can encompass within-species genetic variability in AMF. Hopefully the recognition of this problem by molecular ecologists might lead to the development of novel solutions.

We propose a three-stage strategy towards the development of a working approach to measure more relevant levels of AMF diversity in natural communities. Our proposed approach is certainly not caveat-free but will hopefully provide a framework for future work. The three stages are: (1) to develop a high-throughput DNA-based approach for measuring intraspecific AMF diversity in a target AMF species; (2) to upscale such an approach to be able to encompass intraspecific diversity in key AMF species. Alternatively, develop a database of genetic polymorphism across the Glomeromycota by generating multilocus sequence data for all available isolated AMF taxa from which markers can be developed to target any level of resolution; (3) to use the markers developed in stages 1 and 2 in studies of AMF diversity in natural communities or in experiments designed to investigate the role of different levels of AMF diversity in plant ecology. 
Stage 1

Hart et al. (2015) suggest that other levels of diversity can be addressed in the future, as the genomes of more AMF species are sequenced. However, the development of markers to estimate genetic diversity within a given AMF species is certainly not a trivial undertaking. Information on genetic diversity within AMF populations is extremely limited. Multilocus genotyping has allowed limited measurements of intraspecific variation within one $R$. irregularis population in Switzerland (Koch et al., 2004; Croll et al., 2008). In this case, development of genetic markers was possible because the fungus could be cultured in vitro allowing the extraction of contaminant-free DNA. $R$. irregularis has a large niche range and many isolates of this species exist from around the world. Using reducedrepresentation sequencing (RRS) techniques, such as restriction site-associated DNA sequencing (RADseq) performed on many isolates of $R$. irregularis, it would be possible to build up a database of single nucleotide polymorphisms (SNPs), indels and polymorphic repeat regions that could be used to develop markers encompassing the global genetic diversity in this species. Such an undertaking requires the availability of many isolates of that species but does not rely on existing knowledge about the genome of the species.

\section{Stage 2}

The above-described approach is only practical for an AMF species where a number of isolates already exist. So researchers face the problem of how to scale-up such an approach for more AMF species. As we clearly cannot do this for every AMF species, how can it be done in a practical and pragmatic way?

We propose two different approaches to overcome this problem. The first approach uses existing molecular data on AMF communities to identify which species to target in more detail. The second approach uses phylogenetics and existing isolates of different AMF species to allow the development of different markers to measure AMF diversity at any level of resolution required.

By using existing meta-genomics data, based on rDNA sequences, researchers could identify which AMF taxa are particularly common in a given ecosystem (that is, generalists). This information could then be used to target isolation of those AMF species from within and among a wide range of different sites. Once a collection of many isolates of those species is available, the approach described in stage 1 above (using RAD-seq) could then be used to build up a set of markers that can define the intraspecific genetic variability in each of the multiple generalist AMF species. The markers developed using this approach would allow researchers to measure allele diversity at many loci in environmental samples for many AMF species. The problem with this approach is that isolating target generalist
AMF species and putting them into culture would be extremely time consuming and labour intensive.

A second approach could partially overcome this problem. This involves obtaining DNA from as many existing isolates of AMF taxa as possible that are available in laboratories around the world. Such isolates would represent the phylogenetic range of AMF available in culture. Performing RAD-seq, using Illumina MiSeq technology (Illumina Inc., San Diego, CA, USA), on the DNA of each AMF isolate would generate millions of sequences of up to $300 \mathrm{bp}$. Paired-end sequencing would yield thousands of potential phylogenetically informative sequences of up to $600 \mathrm{bp}$ from each isolate. Such an approach would allow researchers to build up a detailed database of genetic polymorphism across the Glomeromycota phylum. The availability of such data would allow researchers to build up multilocus allele diversity measurements at any level of diversity desired from among AMF genera, among AMF families, among AMF species to within AMF species. Although such an undertaking would be large for one research group, a consortium approach, ultimately generating a publicly accessible database of the sequences is feasible. We believe that such an approach would greatly enhance researchers' capability to measure AMF diversity in environmental samples at any resolution desired.

Stage 3

The availability of a database of SNPs and sequences flanking the SNPs, or other types of polymorphism, would allow the development of a large number of primers for targeting multiple loci. If stages 1 and 2 have been followed, and the first approach in stage 2 is adopted, then this would provide the possibility to measure allele diversity of target AMF species in any sample of DNA recovered from soil or from plant roots.

If the second approach in stage 2 was adopted, then primers could be designed for multiple loci at potentially any level of resolution within the Glomeromycota. By using the whole data set, comprising millions of RAD-seq reads from as many isolated AMF as possible, markers could be developed to estimate among-AMF family genetic diversity in environmental DNA samples. For the development of markers giving a finer resolution, the data set could be restricted to a particular AMF family, genus or species, in order to discover appropriate markers at multiple loci. Finally, where there are enough isolates of a given species, the data set could be restricted to only consider markers that reveal intraspecific variation.

Once polymorphic loci and primers have been identified using data generated from RRS, measurement of multilocus allele diversity in large numbers of environmental samples may not require further high-throughput sequencing. Other more economic, targeted high-throughput approaches exist such as microfluidics-based PCR arrays (such as technology 
developed by Fluidigm, San Francisco, CA, USA) or Nanostring technology for quantitatively measuring frequency of alleles at hundreds of loci in a small number of samples (or a small number of loci in hundreds of samples). High-density Illumina BeadChip technology would allow the screening of the presence and absence of alleles at thousands of loci in thousands of samples; a very powerful tool for screening genetic diversity within many AMF species simultaneously, if the markers existed.

The ability to use such markers to measure intraspecific diversity in an AMF species, or at other resolution, would allow ecologists to make observational and experimental investigations to elucidate whether genetic diversity in a target AMF species influences given processes in plant and ecosystem ecology and also which factors influence intraspecific diversity in the fungus. Finally, the development of markers that can target any resolution in the AMF would finally give researchers the tools to identify which levels of AMF diversity are actually the most ecologically important.

\section{Conclusions}

Although molecular AMF community ecology has yielded exciting new information about distribution of AMF taxa, we emphasize that researchers interested in the ecological role of AMF diversity should consider results of ecological experiments to help define the relevant level of AMF diversity to study. A second step should be to develop molecular-based approaches that will enable the measurement of such diversity. If not, ecologists have the possibility of generating very large amounts of sequence data with limited ecological relevance. If important levels of AMF variation are below the species level then ecologists need to embrace new approaches, despite the enormous challenges. The approaches that we suggest are certainly not caveat-free, and could probably not easily be undertaken by one research group alone. However, they have the potential to help uncover the role of variation in AMF on the ecology and functioning of communities and ecosystems. For this reason, we think that a concerted effort by several research groups, and a true interchange between experimental ecologists and researchers developing molecular tools, is required to effectively elucidate the role of $\mathrm{AMF}$ diversity in natural ecosystems.

\section{Conflict of Interest}

The authors declare no conflict of interest.

\section{Acknowledgements}

We acknowledge financial support from a Swiss National Science Research Project (Bonus of Excellence 310030B_144079) and the University of Lausanne (Cassava
Proof of Concept Project) that allowed the development of these ideas. We thank the editor and three anonymous reviewers for providing constructive comments.

\section{References}

Cotton TEA, Fitter AH, Miller RM, Dumbrell AJ, Helgason T. (2015). Fungi in the future: interannual variation and effects of atmospheric change on arbuscular mycorrhizal fungal communities. New Phytol 205: 1598-1607.

Croll D, Wille L, Gamper HA, Mathimaran N, Lammers PJ, Corradi N et al. (2008). Genetic diversity and host plant preferences revealed by simple sequence repeat and mitochondrial markers in a population of the arbuscular mycorrhizal fungus Glomus intraradices. New Phytol 178: 672-687.

Davison J, Öpik M, Daniell TJ, Moora M, Zobel M. (2011). Arbuscular mycorrhizal fungal communities in plant roots are not random assemblages. FEMS Microbiol Ecol 78: 103-115.

Davison J, Moora M, Öpik M, Adholeya A, Ainsaar L, Bâ A et al. (2015). Global assessement of arbuscular mycorrhizal fungus diversity reveals very low endemism. Science 349: 970-973.

Harrison MJ. (1999). Molecular and cellular aspects of the arbuscular mycorrhizal symbiosis. Annu Rev Plant Physiol Plant Mol Biol 50: 361-389.

Hart M, Aleklett K, Chagnon P-L, Egan C, Ghignone S, Helgason $\mathrm{T}$ et al. (2015). Navigating the labyrinth: a guide to sequence-based, community ecology of arbuscular mycorrhizal fungi. New Phytol 207: 235-247.

Hiiesalu I, Pärtel M, Davison J, Gerhold P, Metsis M, Moora M et al. (2014). Species richness of arbuscular mycorrhizal fungi: associations with grassland plant richness and biomass. New Phytol 203: 233-244.

Hodge A, Campbell CD, Fitter AH. (2001). An arbuscular mycorrhizal fungus accelerates decomposition and acquires nitrogen directly from organic material. Nature 413: 297-299.

Koch AM, Kuhn G, Fontanillas P, Fumagalli L, Goudet J, Sanders IR. (2004). High genetic variability and low local diversity in a population of arbuscular mycorrhizal fungi. Proc Natl Acad Sci USA 101: 2369-2374.

Koch AM, Croll D, Sanders IR. (2006). Genetic variability in a population of arbuscular mycorrhizal fungi causes variation in plant growth. Ecol Lett 9: 103-110.

Krüger M, Krüger C, Walker C, Stockinger H, Schüssler A. (2012). Phylogenetic reference data for systematics and phylotaxonomy of arbuscular mycorrhizal fungi from phylum to species level. New Phytol 193: 970-984.

Mensah JA, Koch AM, Antunes PM, Kiers ET, Hart M, Bücking H. (2015). High functional diversity within species of arbuscular mycorrhizal fungi is associated with differences in phosphate and nitrogen uptake and fungal phosphate metabolism. Mycorrhiza 25: $533-546$.

Moora M, Davison J, Öpik M, Metsis M, Saks Ü, Jairus T et al. (2014). Anthropogenic land use shapes the composition and phylogenetic structure of soil arbuscular mycorrhizal fungal communities. FEMS Microbiol Ecol 90: 609-621. 
Munkvold L, Kjøller R, Vestberg M, Rosendahl S, Jakobsen I. (2004). High functional diversity within species of arbuscular mycorrhizal fungi. New Phytol 164: $357-364$

Öpik M, Vanatoa A, Vanatoa E, Moora M, Davison J, Kalwij JM et al. (2010). The online database MaarjAM reveals global and ecosystemic distribution patterns in arbuscular mycorrhizal fungi (Glomeromycota). New Phytol 188: $223-241$.

Öpik M, Zobel M, Cantero JJ, Davison J, Facelli JM, Hiiesalu I et al. (2013). Global sampling of plant roots expands the described molecular diversity of arbuscular mycorrhizal fungi. Mycorrhiza 23: 411-430.

Pagni M, Niculita-Hirzel H, Pellissier L, Dubuis A, Xenarios I, Guisan A et al. (2013). Density-based hierarchical clustering of pyro-sequences on a large scale - the case of fungal ITS1. Bioinformatics 29: 1268-1274.

Pellissier L, Niculita-Hirzel H, Dubuis A, Pagni M, Guex N, Ndiribe C et al. (2014). Soil fungal communities of grasslands are environmentally structured at a regional scale in the Alps. Mol Ecol 23: 4274-4290.

Senés-Guerro C, Schussler A. (2015). A conserved arbuscular mycorrhizal fungal core-species community colonizes potato roots in the Andes. Fungal Divers; e-pub ahead of print 31 March 2015; doi:10.1007/s13225-015-0328-7.

Vályi K, Rillig MC, Hempel S. (2015). Land-use intensity and host plant identity interactively shape communities of arbuscular mycorrhizal fungi in roots of grassland plants. New Phytol 205: 1577-1586.

van der Heijden MGA, Boller T, Wiemken A, Sanders IR. (1998a). Different arbuscular mycorrhizal fungal species are potential determinants of plant community structure. Ecology 79: 2082-2091.

van der Heijden MGA, Klironomos JN, Ursic M, Moutoglis P, Streitwolf-Engel R, Boller T et al. (1998b). Mycorrhizal fungal diversity determines plant biodiversity, ecosystem variability and productivity. Nature 396: 69-72.

van der Heijden MGA, Martin FM, Selosse M-A, Sanders IR. (2015). Mycorrhizal ecology and evolution: the past, the present and the future. New Phytol 205: 1406-1423.

cc)(1) $\odot$ This work is licensed under a Creative Commons Attribution-NonCommercialNoDerivs 4.0 International License. The images or other third party material in this article are included in the article's Creative Commons license, unless indicated otherwise in the credit line; if the material is not included under the Creative Commons license, users will need to obtain permission from the license holder to reproduce the material. To view a copy of this license, visit http://creativecommons.org/ licenses/by-nc-nd/4.0/ 